\title{
Doing Community: Co-construction of Meaning and Use with Interactive Information Kiosks
}

\author{
Tom Hope, Masahiro Hamasaki, Yutaka Matsuo, Yoshiyuki Nakamura, \\ Noriyuki Fujimura, and Takuichi Nishimura \\ Information Technology Research Institute, \\ National Institute of Advanced Industrial Science and Technology \\ 2-41-6 Aomi, Koto-ku, Tokyo 135-0064, JAPAN \\ \{tom-hope, masahiro.hamasaki, y.matsuo, nakamura-y, \\ nori.fujimura, takuichi.nishimura\}@aist.go.jp \\ http://www. aist.go.jp/
}

\begin{abstract}
One of the challenges for ubiquitous computing is to design systems that can be both understood by their users and at the same time understand the users themselves. As information and its meaning becomes more associated with the communities that provide and use it, how will it be possible to build effective systems for these users? We have been examining these issues via ethnographic analysis of the information and community supporting system that we have developed and employed at conference events. This paper presents initial analysis and suggests greater focus on the interaction between members of micro-communities of users in future ubicomp research.
\end{abstract}

\section{Introduction}

As researchers and developers of ubiquitous computing, we continue to build on the notion of a world where computing systems 'disappear' into the background of social life, seamlessly providing greater abilities for their users to interact with the environment and each other $[1,2]$. This brings with it a responsibility for those involved in the development process to understand the contexts within which the technology is to be used, the users themselves, and, as such, requires knowledge of where similar technology is already in use. CSCW has produced research on computing in work settings, and it can be argued that ubiquitous computing is already a reality for many people in other environments. This is particularly the case in cities, where research is increasingly performed on the challenges of linking infrastructure to front-end sensors and devices, and their associated social systems $[3,4]$. From the perspective of industrial design, it seems difficult to suggest that ubiquitous technology as continued to be envisaged by many in the Ubicomp community can ever exist in the truly 'disappearing' sense. All technology is 'in the world' and humans interact with it as such. It has been suggested $[5,6]$ that technology becomes ubiquitous not because it is physically unseen, but because it has been absorbed into social interaction sufficiently enough that it no longer stands out as being unusual, i.e. being invisible in use. 
Though clearly some infrastructure is largely hidden from view, when we interact with it via interfaces (whether in the form of devices or various sensors and their feedback effects), in other words, when it becomes an object or collection of objects, it becomes visible and exposed. Socially, infrastructure and its associated devices, as with any technology, must become incumbent with meaning when developed, designed and used [7]. A hope of Ubicomp must therefore be to work with these meanings and minimize discordant effects on users of the technology, while recognizing the influence of design processes. Consequently, viewed within a framework of social computing [8], we can assert that technologies can be considered to be ubiquitous when they provide useful functions and at the same time do not cause stoppage or unnecessary difficulties in the social interaction of their users. Using this definition as a basis, this paper presents some analysis of real-world usage of the conference-support system that we have developed. In the rest of this section, we introduce the background to the study and its relevance to ubiquitous computing research. Section 2 introduces some related work, followed by a description of our system, UbiCoAssist, in section 3 . Section 4 presents the analysis of qualitative data of the system in use at two different events. In the light of the findings, we suggest some considerations for future development of Ubicomp at the end of the paper.

\subsection{Community}

In social research, notions of 'community' have always been central. Many of the early sociological studies examined the changing nature of society with reference to community, often mourning or celebrating the demise of a traditional communal way of life [9]. More recent work continues to emphasise the binding nature of community politically and socially, continuing in the same vein to discuss it in relation to its relative (in)stability and (im)permanence [10]. The impact of information and communication technologies, which reduce the need for face-toface interaction, suggest that a sense of community can be gained in the virtual world [11], but space remains for further research into real-world community practices. This is evidenced by recent work on computer-supported cooperative work and ubiquitous computing systems. Many of these studies continue to deploy systems into pre-existant groups, grounded in physical space, and evaluate their designs based on the acceptance or rejection by those groups. Brignull et al, for example, introduced a shared interactive display into a community of high school students, noting both how the space affected use and suggesting that interactive applications should be designed so that "the community can adapt to their own activities" [12]. The sense appears to be that these communities or 'communities of practice' [13] can be designed for, and that by doing so, the technologies will disappear into everyday usage, thus becoming ubiquitous.

We began our own research aiming to support the communities that attend conferences and other similar events. UbiCoAssist therefore contains several concepts of community within its design. Firstly, there is the relatively abstract notion of a community of researchers, some of whom will attend the same events. This is concretised by perceiving of this community in terms of social networks, 
and then further solidified by the members physically attending conferences in the same locations. During the development and analysis of the system, we began to use the term 'micro-community' to denote the small groups of event participants who gather around the kiosks and use them to develop their social networks. The use of this term enables us, like the users, to focus on both the social network and the physical interaction simultaneously.

\subsection{Ubiquitous Kiosks and IC Cards}

While it is clear that multiple handheld technologies are integrated into everyday life for many-mobile phones, PDAs, wireless internet and are obvious examplesmuch of the ubiquitous computing presently in place outside of the laboratory remains geographically fixed. One such example of already existent ubiquitous technology is the information kiosk. In our research, we define such a kiosk as a terminal, located in public or semi-public space, from which users can gain information interactively. This information is often contextually oriented, based on the users identities and previous interactions with the system, and the location of the kiosk itself. Examples include museum information interfaces, interactive street maps, and shopping mall/airport guides. A defining feature of these kiosks is that they are accessible to more than one person. Though one user may control the interface itself, other individuals are able to view the information at the same time, forming groups around the kiosks. We can envisage that many of the functions of these kiosks will move onto mobile devices, but the social affordance of group viewing suggests that there will be a continued need for larger interfaces around which small groups of people can interact.

In addition to these kiosks, IC cards ('smart cards') continue to be taken up by multiple segments of society, as more and more devices and systems are being developed that utilize their relatively cheap and convenient properties. IC cards have recently become a popular alternative to travel fare systems [14] and electronic wallets. In a sense, we can see an IC card as a tangible bit [15], it is the tangible device-a card with information processing functions-of larger information systems. These are therefore tangible interfaces that are already well used in the real world. These cards have rules of use, in the sense that we generally use them in ways appropriate to their function, but this use may vary according to the place, including the country, where they are used. As kiosks, readers and cards become common ways of obtaining and providing information, it is important to understand the potential effects on social interaction. The intersection between these technologies, forms of which are already widely used, is therefore the foundation of our study. How will people understand and make use of interfaces, which directly aim to involve them in the sharing of relationship data? 


\section{Related Work}

\subsection{Kiosks, Tables and Public Displays}

Development continues into making information kiosks more contextually sensitive. Intelligent kiosks [16], for example, aim to provide information in a way that is appropriate for the user. Work has been conducted on developing multimodal information kiosks that recognize who is speaking to it according to facial orientation and gaze $[17,18]$. These suggest that potential exists for extensions of our own work, into the information kiosk as a ubiquitous technology with continuing relevance. The emphasis on interface dynamics, such as in developments of information kiosks as embodied conversational agents [19], also influences the following paper. There is a continuing interest in developing multi-user displays and surfaces, which enable connection between users both publicly and in private [20]. The present work, therefore, lies at the intersection of ubiquitous computing, information design and research into conversation structure and sense-making $[21,22]$ and on a broader level, what Dourish and Button [23] have called 'technomethodology'.

Essential to understanding our current research, is the fact that an information kiosk usually provides information specific to the area in which it is physically located. If it is a map, for example, it will show the location of the kiosk (often denoted as 'you are here'). As a kiosk it is therefore relatively immobile, thus users must themselves be located next to it in order to put it to use. Other potential interfaces have similar features. Tables, for example, arguably remain the most important collaborative spaces, and signify the physical meeting of individuals. This feature-the propensity for people to be physically present together at the object-provides unique opportunities for interaction, which may be lost should the interface be of a different type. In this vein, related work exists in tabletop interface research. One example is Ubitable [24], a system to facilitate collaboration on a tabletop interface. Ubitable enables users to choose where to sit and assigns that seat to them before engaging in interaction with the other user. In this case the space is organized prior to interaction due to the positioning that the interface affords. Where our research in this paper differs, is in the seemingly ad-hoc nature of arrangement around a kiosk-type interface and the possibilities that this brings to interaction with the system. A similar 'loose' arrangement was afforded by the study by Brignull already mentioned $[25,12]$.

\subsection{Conferences and Similar Spaces}

Conference spaces are interesting for research in ubiquitous computing as they are bounded areas with events that occur in limited time periods. This, in combination with the desire to give and receive information, makes fertile ground for testing devices and systems in development. Related research in this field is work on augmenting spaces with various screens and displays. One such development is in proactive displays [26] that sense and react to nearby people. The work that 
most directly connects with our own is the installation of communityware at museums and conferences by Sumi and Mase [27]. In this, as with our own system, community awareness is central, providing opportunities for users to know of others with related interests and provide contextually relevant information. We have therefore also been interested and influenced by developments in mobile sensors [28] and possibilities for combining kiosks and wearable computing in event spaces [29], which may increase awareness.

\section{System Design and Implementation}

UbiCoAssist, forms part of a larger set of technologies developed to support users of conference spaces or other events. UbiCoAssist aims to give support by providing relevant information and allowing users to build and strengthen social relationships before, during and after the event. It consists of web-mining on top of which sits a social network and conference scheduling browser, and a kiosk-based interface. Using the system, a user can find research topics that another researcher is exploring or researchers with whom they are working. In the scheduling part, a user can register interesting presentations (papers, demonstrations and posters) and explore recommended presentations and other researchers.

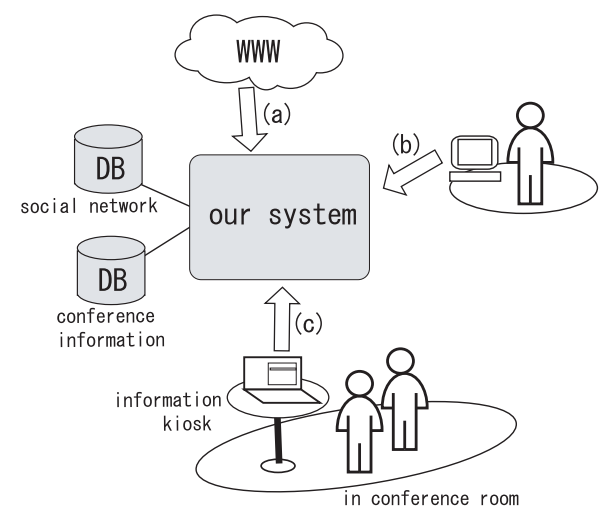

Fig. 1. (a) The system extracts social networks from the web. (b) Users use an online timetable and register their social networks. (c) They can view mutual social relationships. Information kiosks capture their interaction.

Figure 1 presents a system overview of UbiCoAssist. Users can access it via $\mathrm{PC}$ or via one of the information kiosks at the conference site.

Figures 2 and 3 depict an information kiosk. We deployed several of these kiosks in the conference spaces, each with slightly differing designs. Some kiosks had larger screens than others (e.g. a plasma screen, a large projector screen and differing sizes of PC), but all included the same elements and all were positioned to enable the greatest visibility and access. Each information kiosk has two or 


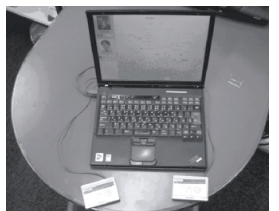

(a) information kiosk

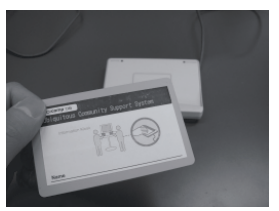

(b) IC card

Fig. 2. Information Kiosk and IC Card

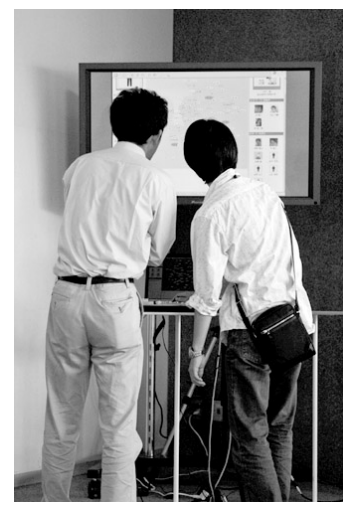

Fig. 3. Two Users at an Information Kiosk.

three commercially available IC card readers [30] and one screen to view and interact with the application. When a single participant puts an IC card on the card reader, they can log in to the system directly. When two or more users log in with their cards at the same kiosk, the action logs them in and presents them with a large social network diagram of their joint social network.

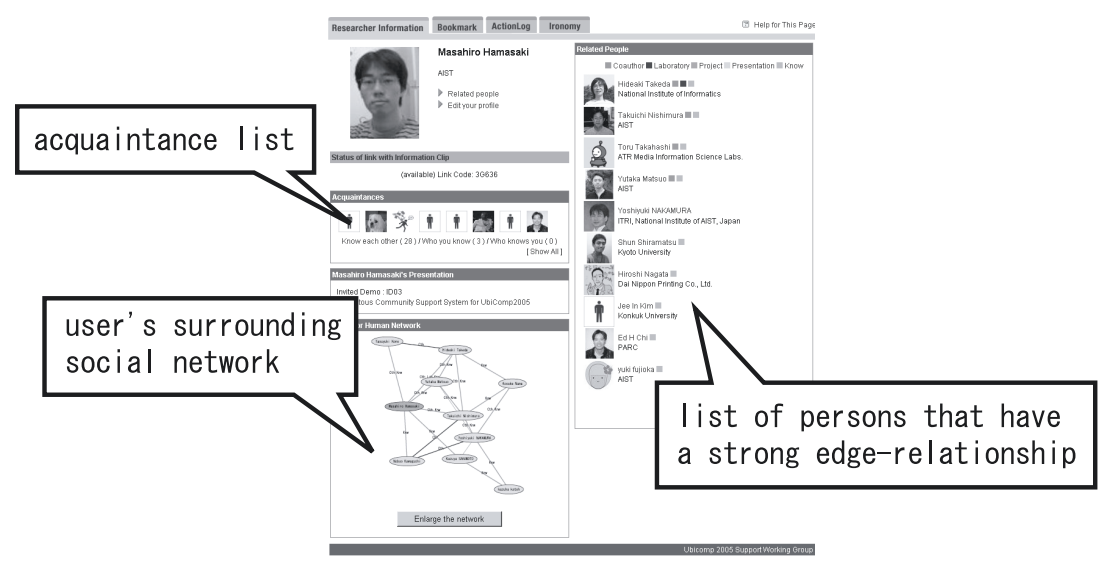

Fig. 4. Mypage

Figure 4 shows a screenshot of Mypage, the personal portal page of UbiCoAssist that users see when they log in. A personal acquaintance list is located in the middle of the left side. The right side consists of a list of authors and other users that have a strong edge-relationship in the social network. The individual user's surrounding social network is shown at the lower left of the page. 
Three methods to Extract Social Networks. UbiCoAssist has three methods to extract social networks among participants. The first of these is based on a web mining technique. We call a link extracted by this method a 'Web Link'. The second is a 'Touch Link', which is based on real-world user interaction with the IC card. The last is 'Know Link', based on user interaction on the web system. This latter method is similar to other Social Network Systems [31]. A 'Web-link is extracted from the Web using a web mining technique. We applied the web mining method based on method of Matsuo et al. [32] to extract a social network among all participants at each conference. Here we present a brief description of the method and its modifications for our system. The simplest approach is to measure the relevance of two nodes based on the number of retrieved results obtained by a search engine query. For example, assume we are to measure the relevance of two names: "Vannevar Bush" (denoted as X) and "Ted Nelson" (denoted as Y). We first address a query "X and Y" to a search engine and get documents including those words in the text. In addition, we make two queries "X" and "Y", and get b1 and b2 documents. The relevance of "Vannevar Bush" and "Ted Nelson"' is approximated as the Overlap coefficient [33]

$$
\operatorname{rel}(x, y)=\operatorname{Overlap}(X, Y)=\frac{\sharp(X \cap Y)}{\min (\sharp(X), \sharp(Y))}
$$

For example, if a is divided by $\mathrm{b}$, the $\operatorname{rel}(\mathrm{x}, \mathrm{y})$ represents the relevance of nodes $\mathrm{x}$ and $\mathrm{y}$.

More than one person can have the same family and given name. To alleviate this problem, one study suggested [32] adding affiliation to the query. However, our aim in developing this system is obtaining social networks in order to support the participants of particular events, to date, these being the community of users and researchers at conferences. Therefore, instead, we add to the query a keyword that is related to the specific community. We use the names of central figures with the above-mentioned method to select such keywords (which we term 'community keywords'). A keyword that is connected closely with central figures of a community and is relatively uncommon on the web serves well as a community keyword.

We discriminate between relationships by consulting retrieved page contents and applying classification rules [32], and define labels for each relationship as follows:

- Coauthor: Coauthors of a technical paper

- Lab: Members of the same laboratory or research institute

- Project: Members of the same project or committee

- Conf: Participants of the same conference or workshop

Using this method UbiCoAssist aims to build a foundation network that includes some information about types of relationships, which users can view and modify at the event with kiosks and their own computers.

A 'Touch-link is registered by users via an information kiosk. Users can see social networks among them when two or three participants use the information 
kiosk together. At this point, the social-tie "We meet and see our social network together" is added to UbiCoAssist automatically. These actions in the real world also create additions of a know-edge on the network.

Users on the web system can register a 'Know-link'. This creation of personal acquaintance lists ("I-know" and "I'm-known-by" lists) is a basic function of the system, providing power to the user to add others to their network via a PC. A user can make an addition to the "I-know" list when that user finds an acquaintance on paper or session pages, or from authors and other users listed in order of calculated weight. At that time, the acquaintance is also added to the acquaintances "I'm-known-by" list. These actions are also additions of a know-edge.

\subsection{Applications with the Social Network}

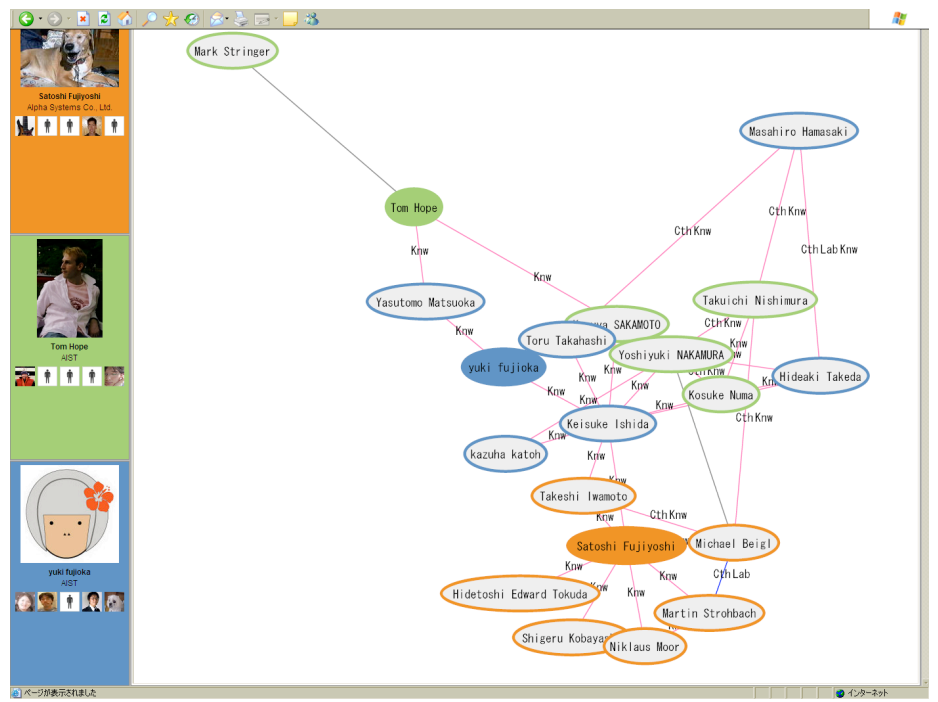

Fig. 5. Three users logged into a kiosk together can view their joint social network

UbiCoAssist displays a social network among users when they use an information kiosk together (Figure 5). Colors around the users photographs to the left of the network diagram refer to the color of the IC card reader they used to log in. The network display is animated: users can drag nodes (names of individuals on their networks) around the screen, further modifying its appearance.

The system additionally has a person (authors and participants) page, session page, and a paper page. Each page shows a surrounding social network and users can view those associated persons. The person page shows a user-egocentric social network. The session page depicts a social network among authors whose 
papers are presented in a session. The paper page shows a social network among authors and users who are interested in that paper. There is also a paper search, which allows users to find the social network organization around inputted keywords.

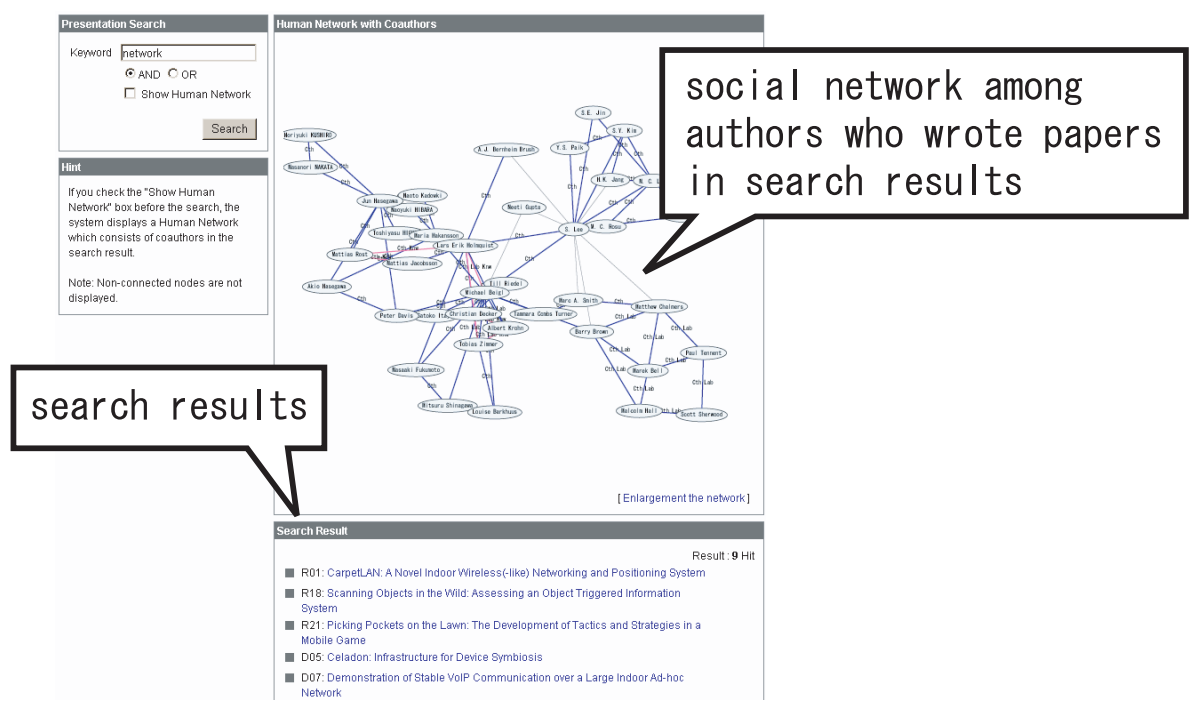

Fig. 6. Results of a paper search

\section{User Study: Building Meaning in Social Network Relationships}

We demonstrated and tested the current version of UbiCoAssist at two conferences in 2005: JSAI $2005^{1}$ and UbiComp 2005. The conferences were in the research field of computing, but the participants of the first were mainly Japanese, with the second being an international conference. Both, however, were held inside Japan and so there were also many Japanese members at UbiComp 2005. At each conference several video cameras were arranged around one kiosk, deemed to be one of the most accessible in the space, while ethnographic observation [34] was done of users of all the kiosks. ${ }^{2}$ Signs were displayed next to the kiosk to inform users that video was taken for use in developing the system and a privacy

1 Japan Society for Artificial Intelligence Annual Conference

2 In much the same sense that 'community' has proved to be a difficult term to pin down, we adopt a broader understanding of ethnographic research than is often used in Ubicomp work. While traditionally, ethnography focusses on relatively longterm observation of members of a social group, here the formation of community in temporary spaces is the topic of study itself. 
agreement explanation was in the system's accompanying leaflet. Additionally, some hand-held video data was collected along with many still photographs of use. Consequently data was gathered in the form of video, photographs and fieldnotes, along with informal on-site interviews. The use of strategically positioned video cameras and all-day recording to digital media allowed detailed post-event analysis to be done of users interaction with the system and with each other in the conference space. The system also recorded an access log of users' kiosk usage. Figure 7 shows the kiosk usage in one hour blocks during September 13th at UbiComp 2005. We analyzed the recorded data using both video and access logs from each day.

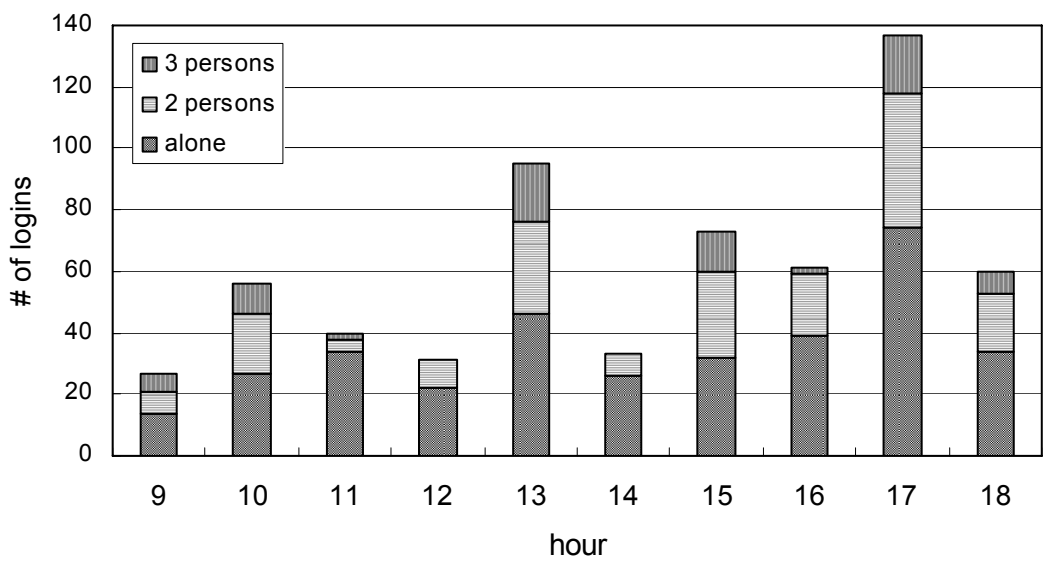

Fig. 7. Number of logins at the kiosk during one day of conference

All social interaction takes place within physical constraints and over definable periods of time. As an analytical strategy, we have found it beneficial to distinguish between the spatial and the temporal aspects to interaction, though in reality these are of course inseparable. Doing this is enabling us to get to grips with the usability issues, and interaction between members, and the methods of sense-making that users used in their interaction with UbiCoAssist.

\subsection{Space and Access}

At each conference, the web-based side of UbiCoAssist was set up online and was running prior to the actual event. Conference participants who registered with the system via their own PCs received passwords via email in order for them to be able to access the system and use its scheduling and network functions. These users were therefore familiar with the browser interface when they first encountered it at the events. However, initial reactions to the information kiosk system were unexpectedly troubled for many users. This was due to several 
reasons, all related to the physical space in which the kiosk and users were located, and the form of the kiosks themselves.

The first issue for users arose when they entered the conference building. A social event suggests expected behavior of participants, but there is also an assumed spatial arrangement, which can cause confusion if it does not fit with expectations. The conferences had registration desks where participants could collect their conference programs, proceedings and identification badges. At the first conference, each person was given an IC card at this desk and then they were directed to the location of the kiosks. The second conference, however, was arranged so that they could register their own (or supplied) IC card with UbiCoAssist at the kiosks themselves, thus allowing them to use any device with a unique ID (including, for example, some mobile phones containing smart chips) if they wished. However, users found it confusing why the kiosks were not located at the same point as the registration desk. The action of 'registering', whether for the conference or for UbiCoAssist, was expected to occur in one location.

It should not be surprising that users on their initial encounter with the system made sense of it according to prior understandings of conferences. UbiCoAssist is, after all, a system that supports conferences and similar events. But it also points to the fact that the space and its associated systems sensors (in this case card readers) are given meaning by the social context in which they are set. This denotes an important difference between space and place [8], and one that will affect the implementation of the system in other types of settings.

Once users were at a kiosk they were confronted with another problem, namely, how to use the IC cards. Users who had not yet registered the card to their identity in UbiCoAssist were required to do so at a kiosk. In order to do this, they needed to place a card onto the reader and leave it there. Removing the card would log them out of the system, partly as a security measure to avoid individuals leaving the kiosk while still logged in and thereby allowing their data to be modified by other participants. This action-placing the card on the reader and leaving it there-was frequently troublesome. Users would place the card and quickly remove it, looking at the screen only to discover that they were not logged in as they had expected. They would repeat the action and receive the same result. The problem was not rectified when more than one user logged in, as they would encounter the same. This can be explained by the affordances of IC cards.

Though not a sensor which requires no action on behalf of the user, IC cards are already a pervasive technology. Due to the limitations of card and sensor, they must be in the correct range of each other in order to function. There are many ways to use a card, and consequently to the user there is no use intrinsic to the object itself. Nevertheless, many of the conference participants use similar types of cards in their daily lives and, as was seen at the conference, this affected their use with the information kiosks. The cards that we use frequently, and that allow access to spaces, are usually used with a brief swiping motion. Keys, more often than not, are inserted upon entry and removed, as are credit cards and tickets. But not all cards are used like this, credit cards being a good example, 
so why did users, upon a first failure to log in, not switch to this other method of use?

The video data provided one explanation in the form of collaborative interaction, as users would negotiate with each other the meaning of the problem. Essentially, it emerged that the human error of not logging in correctly was often suggested to be a system error. As soon as one user stated this, the other user would soon agree and further attempts at using the system together would be abandoned. It may be that the members of the conferences, themselves being researchers and developers in computing, were expecting system errors. This may also have been the reason why some participants were unsure which other devices they could use to access UbiCoAssist. There were several occurrences of individuals asking how to access with their PDAs, mobile phones or indeed via the systems on display by other non-related demonstration teams in the conference space. Being somewhat contrary to the findings of Williams et al [35], who observed users seeking to understand their system, SignalPlay, in terms of individual components, these users of UbiCoAssist were attempting to understand the whole system and fit different devices into it.

The interface objects, then, and the space where they were used, did not in themselves inform users of the methods for their use. Clearly, there were expectations of how the system would behave, but when these were not fulfilled, users experienced difficulties.

\subsection{Time and Identity}

A significant characteristic of a conference or similar event is that it exists only for a specific time, and therefore systems of ubiquitous technology must deal with large aggregates of new users, who only have a relatively short time within which to develop appropriate practices. This time itself holds some order in the interaction of users. Failure to log in to the system the first time did not prevent the user from trying again. However, if the failure had been in a group, such as in the perceived system error described above, the user may attempt to access the system at a later time, individually. Conversely, we observed users who failed individually, trying again later, but this time in a group. The fact that these individuals are professional researchers of computing may have played no small part in the aversion to lose face [36], and a group could therefore provide, or not provide, mutual support. However, the fact that users could view the networks of others and see, therefore, if they had touch-links or not, may have served as a prompt.

We know from studies of conversation that interaction is ordered in a sequential manner [21]. This temporal order defines to some extent the meaning that is attributed to UbiCoAssist by its users. Figure 8 illustrates this with a transcribed excerpt from a conversation between two users of the system, A and $\mathrm{B}$, who were viewing their joint social network. Both users had logged into the system before, using their IC cards, but this was the first time they had been at the same kiosk together. 


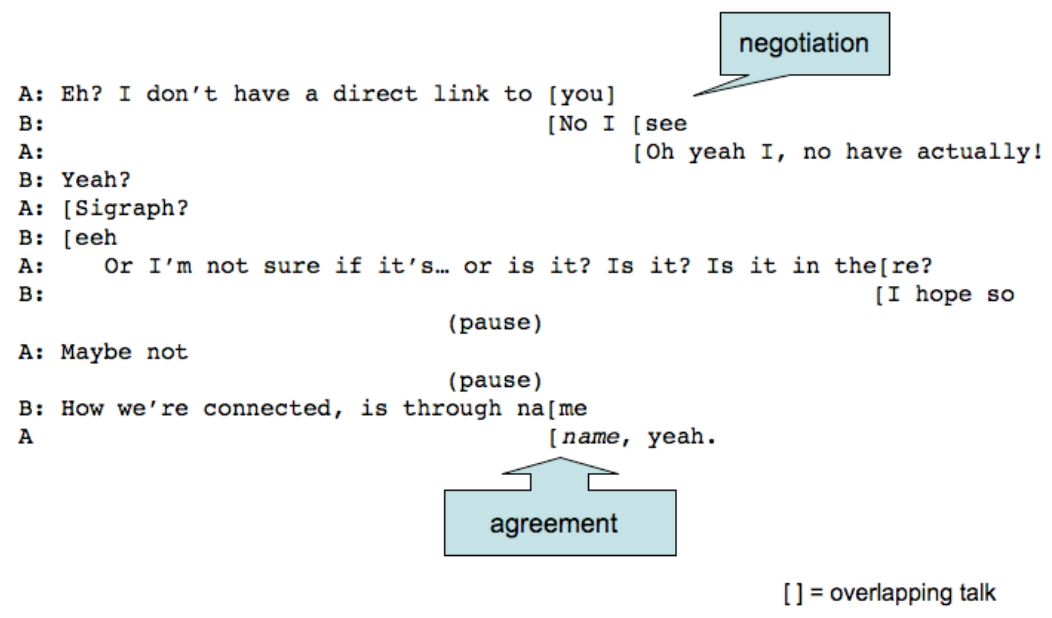

Fig. 8. Negotiating the network

As illustrated with the extract, the sequential order of interaction around the kiosk has possibilities to affect the understanding of the network. Some negotiation took place in the conversation, with neither party firmly stating the connection between them until the last line of the extract. The corresponding social network actually contained several linked people between them, so the negotiation may have been referring to who was thought to be the most direct, or influential link. In observing these and other users, it is apparent that the understanding of these links is very subjective. Figure 8 shows, therefore, how important the sequence of talk is to making sense of the visualized community. If either A or B had firmly claimed a relationship first, it could have changed the course of the rest of the interaction, and therefore the sense of what relationships the social network was representing.

\subsection{Doing Community}

Information kiosks demand that users be standing near or next to them in order to be used. This requirement in and of itself provides a context in which groups can form with similar goals and interests. Much like a table or other communal object, there is the possibility for members of already existent communities to gather together, but we also found that small temporary communities can be created there. The physical gathering of users affords awareness [37] of the activities of others, which enables monitoring of their action with the system, and therefore can lead to mutual orientation to similar practices.

UbiCoAssist provides a visual diagram of a community of participants at an event, but, as powerful as the web-mining algorithm is, in itself it does not guarantee a perfectly correct representation. In fact, what we found was that its perceived imperfections created spaces for further interaction between users, as 
shown above in Figure 8. The perceived system errors described in section 4.1 occasionally did the same thing, but though we can attribute this as associated with the occupation of users, the former should feasibly be the case for any user at any event. Of course, users actions were not solely oral as suggested in this extract, but were wholly embodied, incorporating gesture, gaze, bodytorque $[38,39]$ and an understanding of social behavior (Figure 9). An example can be found in the relatively ordered manner in which some of the Japanese participants allowed particular members of their groups to access the interface before others. These members often held more influential positions on the social network diagrams, but as this was not always the case, further study is required to fully grasp the role that it may play. The least we can say is that this form of granting access to other users was a negotiated action between several members of the groups at the kiosks.

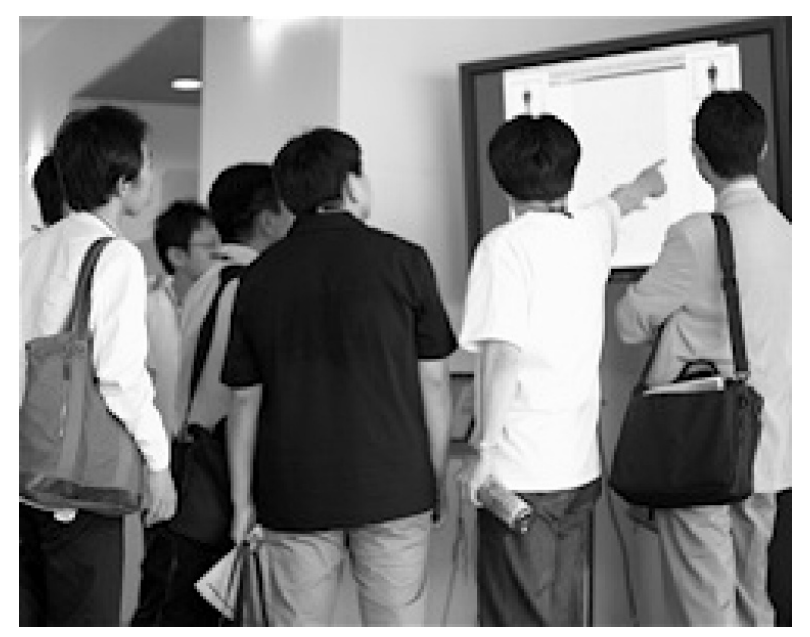

Fig. 9. Gesturing to the Interface

Bodies hold very different meanings when in the setting of social networks and public displays. UbiCoAssist shares elements of both virtual and real-world communities and it was down to the users to negotiate the connections between these worlds at the time of the conferences. At the beginning of the events, the communities as envisaged by the developers of UbiCoAssist were principally virtual, diagrammatic ones, represented as social networks. During the actual events however, real-world communities soon formed. These may arguably be perceived as communities of practice [13], as they encompassed both knowledge of how to use UbiCoAssist, experience of its use, and an ongoing practice of using it in ways appropriate to each other, but seen in that frame it is remarkable how quickly the community formed and therefore perhaps this is not the appropriate definition. We have defined these small groups that form at the conferences 
around kiosks as 'micro-communities', denoting possibly transient interactions between people, but showing potential to become stronger connections in the social networks at future events.

\section{Conclusions and Future Development}

We have seen in this paper that it is not possible to rely purely on the physical form of interfaces and spaces in order to create smooth use of kiosk-based information and networking systems. In spite of advanced planning, usability issues quickly arise. Instead, if we are to produce technology that is invisible in use, it is essential to gain knowledge of the social understanding of the use and meaning of these systems. We saw that individuals when encountering the technology collaboratively work to understand its possible use and subsequently give it meaning in the way that they use it. With UbiCoAssist, this co-construction of social network, micro-community and the meaning of the system itself occurs in the temporally organized interaction around the kiosk. We can assert that other systems and settings where multiple users congregate will also be made sense of in a similar way, with the interaction between users again playing an important role. Though social expectations in some ways structured the access and use of the kiosks, it was negotiation between users at the time that gave meaning to its use.

It is clear that UbiCoAssist can be improved by addressing further the issues raised in this paper. While the system as it is does allow room for 'dissent'-i.e. users are able to modify their network if they disagree with it, or at least not take part in the system save for their name being used as a node on a social network - it may be possible to give an even greater sense of security to users developing ways to 'break' links or disassociate oneself from others. Nevertheless, the system as it is provides an effective platform for social engagement with others, within which negotiation can take place. The ability given to users to construct networks as they perceive them also negates to an extent some of the problems of building the initial social networks around community keywords (which some may disagree with). These keywords and the web-mining inherently structure the networks before the conferences and we are consequently continuing to explore these issues.

Many of us in the field of ubiquitous computing have an image in mind when we work on the development of our technologies. We conceive of products, and develop systems with ideas of an eventual end-user community. One of the dangers in this process is that it is easy to use the conception of community as a gloss - a categorization shorthand to describe what is actually a multitude of very different individuals. When faced with the task of understanding their own community, as was the case with the users of UbiCoAssist and its social network, it soon became clear that it was not as easy for them as one might have expected. There were times when, clearly, interaction with both the system and between users themselves was difficult, conversation became stilted and exasperation emerged. Yet, certain things did help combat these difficulties. Firstly, 
the ability to modify the social network in some way helped by giving control to the user to adjust the results of the web-mining. Secondly, the sequential manner of interaction enabled users to collaboratively construct a sense of what the network - and the individuals represented on it - meant to them. Thus, contextual information in the form of the social network became more accurate. This combination of automation and user control may improve the effectiveness of similar systems.

Community was, then, 'done' by the participants of the conference when they used UbiCoAssist, rather than simply existing before the event. This doing of community holds important ramifications for the development of ubiquitous computing that hopes to use contextual information about users identities or characteristics collected prior to use, as it suggests that real-time sensing of how micro-communities are forming and the practices they engage in will be a necessary addition.

Acknowledgements. This research has been supported by NEDO (New Energy and Industrial Technology Development Organization) as the project ID of 04A11502a.

\section{References}

1. Weiser, M.: The computer for the 21st century. Scientific American 265(3) (1991) 66-75

2. Satyanarayanan, M.: Pervasive computing: Vision and challenges. IEEE Personal Communications (2001) 10-17

3. Mainwaring, S.D., Chang, M.F., Anderson, K.: Infrastructures and their discontents: Implications for ubicomp. In: Ubicomp 2004. (2004) 418-432

4. Star, S.L.: Infrastructure and ethnographic practice: working on the fringes. Scandinavian Journal of Information Systems 14(2) (2002) 107-122

5. Kuniavsky, M.: What's invisible technology? no, really. Workshop on situated ubiquitous computing at Ubicomp 2005 (2005)

6. Heer, H., Khooshabeh, P.: Seeing the invisible. In: Workshop on Invisible and Transparent Interfaces at AVI 2004, ITI Workshop, part of AVI 2004 (Advanced Visual Interfaces) (2004)

7. Suchman, L.: Working relations of technology production and use. ComputerSupported Cooperative Work (CSCW) 2 (1994) 21-39

8. Dourish, P.: Where the action is: the foundations of embodied interaction. MIT Press, Cambridge, MA, USA (2001)

9. Tonnies, F.: Community and Civil Society. Cambridge University Press, Cambridge (2001)

10. Delanty, G.: Community. Routledge, London (2003)

11. Wellman, B.: The persistence and transformation of community: From neighborhood groups to social networks (2001)

12. Brignull, H., Izadi, S., Fitzpatrick, G., Rogers, Y., Rodden, T.: The introduction of a shared interactive surface into a communal space. In: CSCW '04: Proceedings of the 2004 ACM conference on Computer supported cooperative work, New York, NY, USA, ACM Press (2004) 49-58 
13. Wenger, E.: Communities of practice: learning, meaning, and identity. Cambridge University Press, Cambridge, U.K. and New York, N.Y. (1998)

14. JR Suica: http://www.jreast.co.jp/suica/index.html (2006)

15. Ishii, H., Ullmer, B.: Tangible bits: towards seamless interfaces between people, bits and atoms. In: CHI '97: Proceedings of the SIGCHI conference on Human factors in computing systems, New York, NY, USA, ACM Press (1997) 234-241

16. Christian, A.D., Avery, B.L.: Speak out and annoy someone: experience with intelligent kiosks. In: CHI '00: Proceedings of the SIGCHI conference on Human factors in computing systems, New York, NY, USA, ACM Press (2000) 313-320

17. Bakx, I., van Turnhout, K., Terken, J.M.B.: Facial orientation during multi-party interaction with information kiosks. In: INTERACT. (2003)

18. Mäkinen, E., Patomäki, S., Raisamo, R.: Experiences on a multimodal information kiosk with an interactive agent. In: NordiCHI '02: Proceedings of the second Nordic conference on Human-computer interaction, New York, NY, USA, ACM Press (2002) 275-278

19. Cassell, J., Stocky, T., Bickmore, T., Gao, Y., Nakano, Y., Ryokai, K., Tversky, D., Vaucelle, C., Vilhjlmsson, H.: Mack: Media lab autonomous conversational kiosk. In: IMAGINA '02, Monte Carlo (2002)

20. Greenberg, S., Rounding, M.: The notification collage: posting information to public and personal displays. In: CHI '01: Proceedings of the SIGCHI conference on Human factors in computing systems, New York, NY, USA, ACM Press (2001) $514-521$

21. Sacks, H.: Lectures on Conversation. Blackwell Publishers, Oxford (1995)

22. Garfinkel, H.: Studies in Ethnomethodology. Polity Press, Cambridge (1996)

23. Dourish, P., Button, G.: On "technomethodology": Foundational relationships between ethnomethodology and system design. Human-Computer Interaction 13(4) (1998) 395-432

24. Chia, S., Everitt, K.: Ubitable: Impromptu face-to-face collaboration on horizontal interactive surfaces. In: Proceedings of Ubicomp 2003, Ubicomp (2003)

25. Brignull, H., Rogers, Y.: Enticing people to interact with large public displays in public spaces. In: INTERACT. (2003)

26. McCarthy, J.F., Nguyen, D.H., Rashid, A.M., Soroczak, S.: Proactive displays and the experience ubicomp project. SIGGROUP Bull. 23(3) (2002) 38-41

27. Sumi, Y., Mase, K.: Supporting the awareness of shared interests and experiences in communities. International Journal of Human-Computer Studies 56(1) (2002) $127-146$

28. Nakashima, H.: Cyber assist project for situated human support. In: Proceedings of the Eighth International Conference on Distributed Multimedia Systems. (2002) $1-3$

29. Dey, A.K., Salber, D., Abowd, G.D., Futakawa, M.: The conference assistant: Combining context-awareness with wearable computing. In: ISWC '99: Proceedings of the 3rd IEEE International Symposium on Wearable Computers, Washington, DC, USA, IEEE Computer Society (1999) 21-28

30. Sony Corp., FeliCa: http://www.sony.net/Products/felica/index.html (2006)

31. Mixi: http://mixi.jp (2006)

32. Matsuo, Y., Tomobe, H., Hasida, K., Ishizuka, M.: Finding social network for trust calculation. In de Mántaras, R.L., Saitta, L., eds.: ECAI, IOS Press (2004) 510-514

33. Manning, C.D., Schütze, H.: Foundations of Statistical Natural Language Processing. The MIT Press, Cambridge, Massachusetts (1999)

34. Hammersley, M., Atkinson, P.: Ethnography: Principles in Practice. Routledge, London (1983) 
35. Williams, A., Kabisch, E., Dourish, P.: From interaction to participation: Configuring space through embodied interaction. In: Ubicomp. (2005) 287-304

36. Goffman, E.: Interaction Ritual: Essays on Face-to-Face Behavior. Doubleday Anchor, New York, NY (1967)

37. Dourish, P., Bellotti, V.: Awareness and coordination in shared workspaces. In: Proceedings of the ACM Conference on Computer Supported Cooperative Work (CSCW'92), Toronto, Ontario, ACM Press (1992) 107-114

38. Lerner, G.: Selecting next speaker: The context-sensitive operation of a contextfree organization. Language in Society 32 (2003) 177-201

39. Schegloff, E.A.: Body torque. Social Research 65 (1998) 535-86 